\title{
A NOVEL EM ANALYSIS OF DOUBLE-LAYERED THICK FSS BASED ON MM-GSM TECHNIQUE FOR RADOME APPLICATIONS
}

\author{
S. Narayan*, K. Prasad, R. U. Nair, and R. M. Jha
}

Computational Electromagnetics Laboratory, Aerospace Electronics and Systems Division, CSIR-National Aerospace Laboratories, Bangalore 560017, India

\begin{abstract}
The EM analysis of double-layered thick FSS structure with low-loss dielectric medium between the FSS layers has been carried out using MM-GSM technique. In this analysis, both evanescent and propagating modes are included that enhances the accuracy of the computation. This method provides less computational complexity in the formulation of FSS structures as compared to other numerical techniques. The cascaded FSS structure shows bandpass response ( $>95 \%$ transmission) over a frequency range from $8.84 \mathrm{GHz}$ to $10.74 \mathrm{GHz}$. It is found that this FSS structure shows very good in-band transmission characteristics and excellent roll-off characteristics outside the band. Further, the dependence of transmission characteristics on the spacing between the FSS layers is also investigated. The optimum bandpass response is achieved for $0.3 \lambda$ spacing between the layers. The proposed FSS structure offers superior bandpass response and structural rigidity required for airborne radome applications.
\end{abstract}

\section{INTRODUCTION}

The cascaded structures of periodic surfaces perforated by either rectangular or circular apertures have been widely used in dualreflector antennas with FSS sub-reflector and hybrid radomes $[1,2]$. The transmission characteristics of cascaded FSS structures are known to have a flatter top with fast roll-off characteristics [3] compared to that of single layer FSS. The dual and multi-band characteristics can be obtained by cascading two or more FSS structures. Several

Received 17 October 2011, Accepted 22 November 2011, Scheduled 30 November 2011

* Corresponding author: Shiv Narayan (shivnarayan@nal.res.in). 
analytical and numerical methods have been developed in the open domain for designing FSS structures. The increased complexity of the frequency response of these structures has impelled the researchers to investigate the multilayered FSS structures. Lee et al. [4] presented a simple formula based on matrix approach for the analysis of cascaded FSS structures. However, this approach is accurate only for large separation between the layers $(d>\lambda / 3)$ at normal incidence. Further, the multilayered periodic structures were analyzed using method of moment (MoM) [5,6] and discrete plane wave spectrum (DPWS) method [7]. In MoM technique, the size of the matrix increases with the increase in number of FSS screens, and hence there is complexity in compilation. Compared to the above mentioned methods, Mode matching-Generalized scattering matrix (MM-GSM) method is well suited for both single and multilayered structures at normal as well as oblique incidence. This method is based on matching of the total mode fields at each junction between the uniform sections of the structure, which enhances the accuracy of computation. Further the EM performance analysis based on MM-GSM technique enables one to compute exact number of propagating and evanescent modes associated with the FSS structure.

Munk [8] had proposed both double-layered and multi-layered FSS structures for radome applications, where the double layer FSS consist of either slotted thin screen embedded in the dielectric medium or slotted thin screens separated by the dielectric medium. The EM analysis of double-layered slotted aperture-type thin FSS was presented in [9] using MoM and integral equation method. In the present paper, the EM analysis of two cascaded rectangular aperture-type thick FSS has been carried out using MM-GSM technique, with low-loss dielectric medium between the FSS layers and inside the aperture region of each FSS layer. The objective of the present work is to design a thick FSS based radome wall configuration, which can provide superior band-pass response as well as structural rigidity required for airborne radome applications.

\section{THEORETICAL ASPECTS}

The geometry of the double-layered FSS is shown in Fig. 1, which consists of two similar rectangular aperture-type thick FSS layers separated by a low-loss dielectric medium of thickness $d$. Here TM polarized plane wave is incident on the surface of FSS Layer 1 from left side at an arbitrary angle $\theta$ and propagates along the positive $z$ direction. The FSS layer has a periodicity $T_{x}$ and $T_{y}$, along $X$ and $Y$-directions, respectively. Here $\alpha$ is the skew angle, i.e., the angle 


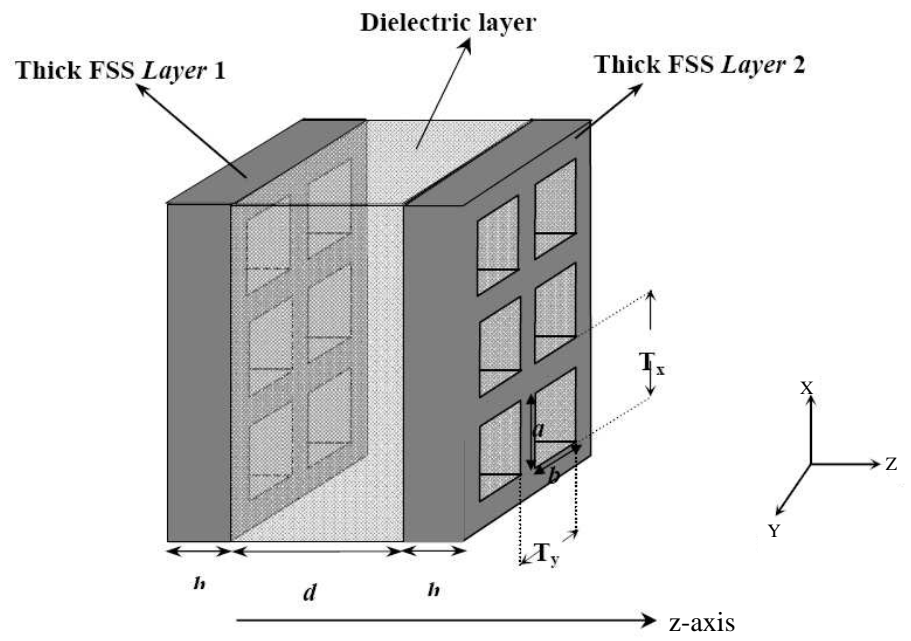

Figure 1. Geometry of double-layered thick FSS structure.

between the directions of periodicities. The dimensions of each unit cell are represented by $a$ and $b$ along $X$ and $Y$-directions, respectively.

The fields on the left side of FSS Layer 1 and right side of the FSS Layer 2, and within the dielectric layer are expanded in terms of Floquet modes with propagation constant $\gamma_{m n}$ and $\gamma_{m n}^{\prime}$, respectively along the $z$-axis as [10]

$$
\begin{aligned}
& E_{T}^{f}(\rho, z)=\sum_{m}\left(a_{m}^{+}+a_{m}^{-}\right) E_{T_{m}}^{f}(\rho) \\
& H_{T}^{f}(\rho, z)=\sum_{m}\left(a_{m}^{+}-a_{m}^{-}\right) H_{T_{m}}^{f}(\rho)
\end{aligned}
$$

where $a_{m}^{ \pm}(z)=A_{m}^{ \pm} e^{ \pm \gamma_{m n} z}$ represent the amplitudes of Floquet modes, and $\rho=x \hat{x}+y \hat{y}$ is the position vector of the unit cell. Here $E_{T_{m}}^{f}$ and $H_{T_{m}}^{f}$ are the transverse components of electric and magnetic fields for Floquet modes, respectively. Subscript $m$ represents the number of Floquet modes outside the structure.

The fields inside the aperture region are expressed in terms of the classical waveguide modes with propagation constant $\beta_{p q}$ as

$$
\begin{aligned}
& E_{T}^{w}(\rho, z)=\sum_{p}\left(b_{p}^{+}+b_{p}^{-}\right) E_{T_{p}}^{w}(\rho) \\
& H_{T}^{w}(\rho, z)=\sum_{p}\left(b_{p}^{+}-b_{p}^{-}\right) H_{T_{p}}^{w}(\rho)
\end{aligned}
$$


where $E_{T_{m}}^{w}$ and $H_{T_{m}}^{w}$ represent the transverse components of electric and magnetic fields respectively. Here $b_{p}^{ \pm}(z)=B_{p}^{ \pm} e^{ \pm \beta_{p q} z}$ represents the amplitude of waveguide modes. Subscript $p$ represents the number of waveguide modes inside the aperture.

By matching the transverse component of electric and magnetic fields at the aperture plane $z=0$, a system of linear equations for waveguide coefficients are obtained by [11]

$$
\sum_{m}\left(A_{m}^{+}+A_{m}^{-}\right) R_{m n}=\sum_{p}\left(B_{p}^{+}+B_{p}^{-}\right) C_{p n}
$$

and

$$
\sum_{m}\left(A_{m}^{+}-A_{m}^{-}\right) C_{n p}^{*}=\sum_{p}\left(B_{p}^{+}-B_{p}^{-}\right) Q_{p q}
$$

where $A_{m}^{ \pm}$and $B_{p}^{ \pm}$represent the mode coefficients for Floquet and waveguide modes respectively. Here $R$ and $Q$ are the power integrals for the free space/dielectric and aperture region respectively, and $C$ represents the coupling power integral between the free space and aperture modes [12].

\subsection{Scattering Matrix Formulation}

Since the power coupled through the cross-polarized TM-TE field interaction is negligibly small, only the co-polarized TM-TM field interaction is considered in the determination of generalized scattering matrix of the FSS structure. The cross sectional view of the double layered FSS is shown in Fig. 2, where $S^{a}, S^{b}, S^{d}$ and $S^{f}$ are the junction scattering matrices of FSS. $P_{1}, P_{3}$ and $P_{2}$ represent the propagation matrices of FSS Layer 1, FSS Layer 2, and dielectric region, respectively. $\mathrm{A}, \mathrm{B}, \mathrm{C}, \mathrm{D}$, and $\mathrm{F}$ are the mode coefficients in the Regions 1,2 , and 3 , respectively. In order to compute the scattering matrix of the proposed double-layered thick FSS structure, the structure is divided into three layers such as FSS Layer 1, dielectric layer, and FSS Layer 2, and four junctions namely air-aperture, aperture-dielectric, dielectric-aperture, and aperture-air. Then the scattering matrices $S^{a}, S^{b}, S^{d}$ and $S^{f}$ are computed independently at the corresponding junctions using MM-GSM technique. Finally, the scattering matrix of double-layered thick FSS is obtained by cascading the scattering matrices of four junctions with the help of propagation matrix of the corresponding layer.

The scattering matrix at the interface $z=0$ is given as

$$
\left(\begin{array}{c}
A_{0}^{-} \\
B_{0}^{+}
\end{array}\right)=\left(\begin{array}{cc}
S_{11}^{a} & S_{12}^{a} \\
S_{21}^{a} & S_{22}^{a}
\end{array}\right)\left(\begin{array}{c}
A_{0}^{+} \\
B_{0}^{-}
\end{array}\right)
$$




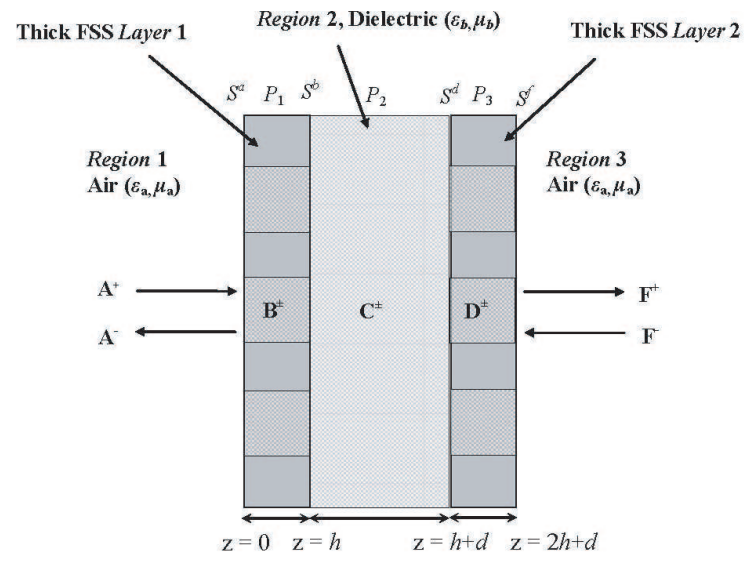

Figure 2. Cross-sectional view of double-layered thick FSS structure.

where the scattering parameters of the matrix $S^{a}$ in the initial set-up of equations are given by [11]

$$
\begin{aligned}
& S_{11}^{a}=-\left(R_{0}+C_{0}^{t} Q_{0}^{*-1} C_{0}^{*}\right)^{-1}\left(R_{0}-C_{0}^{t} Q_{0}^{*-1} C_{0}^{*}\right) \\
& S_{12}^{a}=2\left(R_{0}+C_{0}^{t} Q_{0}^{*-1} C_{0}^{*}\right)^{-1} C_{0}^{t} \\
& S_{12}^{a}=2\left(Q_{0}^{*}+C_{0}^{*} R_{0}^{-1} C_{0}^{t}\right)^{-1} C_{0}^{*} \\
& S_{22}^{a}=\left(Q_{0}^{*}+C_{0}^{*} R_{0}^{-1} C_{0}^{t}\right)^{-1}\left(Q_{0}^{*}-C_{0}^{*} R_{0}^{-1} C_{0}^{t}\right)
\end{aligned}
$$

The scattering matrix at the interface $z=h$ is given as

$$
\left(\begin{array}{c}
B_{h}^{-} \\
C_{h}^{+}
\end{array}\right)=\left(\begin{array}{ll}
S_{11}^{b} & S_{12}^{b} \\
S_{21}^{b} & S_{22}^{b}
\end{array}\right)\left(\begin{array}{c}
B_{h}^{+} \\
C_{h}^{-}
\end{array}\right)
$$

The scattering parameters of matrix $S^{b}$ are given by [11].

The scattering matrices $S^{a}$ and $S^{b}$ are cascaded using the propagation matrix $P_{1}$, the scattering matrix $S^{I}$ is obtained as

$$
\left.\begin{array}{l}
S_{11}^{I}=S_{11}^{a}+S_{12}^{a} P_{1}\left(I-P_{1} S_{11}^{b} P_{1} S_{22}^{a}\right)^{-1} S_{11}^{b} P_{1} S_{21}^{a} \\
S_{12}^{I}=S_{12}^{a} P_{1}\left(I-P_{1} S_{11}^{b} P_{1} S_{22}^{a}\right)^{-1} S_{22}^{b} \\
S_{21}^{I}=S_{21}^{b} P_{1}\left(I-P_{1} S_{11}^{b} P_{1} S_{22}^{a}\right)^{-1} S_{21}^{a} \\
S_{22}^{I}=S_{22}^{b}+S_{21}^{b} P_{1}\left(I-P_{1} S_{11}^{b} P_{1} S_{22}^{a}\right)^{-1} S_{22}^{a} P_{1} S_{12}^{b}
\end{array}\right\}
$$

The junction at $z=h+d$ is the mirror image of the junction at $z=h$. The scattering matrix $S^{d}$ at the interface $z=h+d$ will be the transpose of the matrix $S^{b}$ at $z=h$ interface. Now, the scattering matrices $S^{I}$ 
and $S^{d}$ are again cascaded using propagation matrix $P_{2}$ to form the resultant matrix $S^{I I}$. The scattering parameters are given as

$$
\left.\begin{array}{l}
S_{11}^{I I}=S_{11}^{I}+S_{12}^{I} P_{2} S_{11}^{d} P_{2} S_{21}^{I}\left(I-P_{2} S_{11}^{d} P_{2} S_{22}^{I}\right)^{-1} \\
S_{12}^{I I}=S_{12}^{I} P_{2} S_{22}^{d}\left(I-P_{2} S_{11}^{d} P_{2} S_{22}^{I}\right)^{-1} \\
S_{21}^{I I}=S_{21}^{d} P_{2} S_{21}^{I}\left(I-P_{2} S_{11}^{d} P_{2} S_{22}^{I}\right)^{-1} \\
S_{22}^{I I}=S_{22}^{d}+S_{21}^{d} S_{22}^{I} P_{2} S_{12}^{d} P_{2}\left(I-P_{2} S_{11}^{d} P_{2} S_{22}^{I}\right)^{-1}
\end{array}\right\}
$$

The junction at $z=2 h+d$ is the mirror image of the junction at $z=0$. The scattering matrix $S^{f}$ at the interface $z=2 h+d$ will be transpose of the matrix $S^{a}$ at $z=0$ interface.

Finally, the scattering matrices $S^{I I}$ and $S^{f}$ are cascaded using propagation matrix $P_{3}$ to obtain the final scattering matrix $S^{I I I}$ of the composite FSS structure. The scattering parameters in the final form are given by as

$$
\left.\begin{array}{l}
S_{11}^{I I I}=S_{11}^{I I}+S_{12}^{I I} P_{3} S_{11}^{f} P_{3} S_{21}^{I I}\left(I-P_{3} S_{11}^{f} P_{3} S_{22}^{I I}\right)^{-1} \\
S_{12}^{I I I}=S_{12}^{I I} P_{3} S_{22}^{f}\left(I-P_{3} S_{11}^{f} P_{3} S_{22}^{I I}\right)^{-1} \\
S_{21}^{I I I}=S_{21}^{f} P_{3} S_{21}^{I I}\left(I-P_{3} S_{11}^{f} P_{3} S_{22}^{I I}\right)^{-1} \\
S_{22}^{I I I}=S_{22}^{I I}+S_{21}^{f} S_{22}^{I I} P_{3} S_{12}^{I I} P_{3}\left(I-P_{3} S_{11}^{f} P_{3} S_{22}^{I I}\right)^{-1}
\end{array}\right\}
$$

The above expressions represent the components of generalized scattering matrix of the composite rectangular aperture-type doublelayered thick FSS structure. The transmission and reflection characteristics of the FSS structure are determined using these expressions.

\subsection{Computation of Floquet and Waveguide Modes}

The selection of number of Floquet and waveguide modes for the MM-GSM analysis has an important role towards the accuracy of the solution. For a single layer FSS, the scattering parameters decay exponentially with increasing value of $\gamma_{m n}$ (i.e., for increasing order of Floquet modes $m$ and $n$ ) and layer thickness $h$ as [13],

$$
\left|S_{m n}\right| \sim e^{-\gamma_{m n} h}
$$

For the convergence of solution, all the higher-order Floquet harmonics are included only in $S$-matrix that have magnitude greater than $-22 \mathrm{~dB}$. Here for a given value of $h=0.11 \mathrm{~cm}, \gamma_{m n_{\max }}=$ 4606.5 is obtained. In order to find out the sufficient number of Floquet modes in the numerical calculation, the Floquet modes are arranged in the increasing order of propagation constant $\gamma_{m n}$ and 
corresponding transverse wave number. Then all the Floquet modes having propagation constant value $\gamma_{m n} \leq \gamma_{m n_{\max }}$ are selected and consequently computed the transverse wave number $\tau_{m n}$ corresponding to $\gamma_{m n_{\max }}$. The waveguide modes are calculated using a rule of thumb: the maximum value of the transverse wave number in each region of FSS structure should be same. It is given by

$$
\left|\tau_{m n}\right|_{\max } \approx\left|k_{p q}\right|_{\max }
$$

where $\tau_{m n}$ and $k_{p q}$ are the Floquet and waveguide transverse wave numbers respectively. Similarly, the waveguide modes in the increasing order of cut-off wave number $k_{p q}$ are arranged and then all the waveguide modes having cutoff wavenumber $k_{p q} \leq k_{p q_{\max }}$ are selected.

\section{EM DESIGN ASPECTS AND PERFORMANCE ANALYSIS}

For the EM performance analysis of double-layered thick FSS structure, FSS Layer 1 and FSS Layer 2 are considered to be identical. Here the optimum dimensions of each FSS layer are evaluated at the frequency $10 \mathrm{GHz}$. The thickness of each FSS layer is $0.11 \mathrm{~cm}$. The optimum dimensions of rectangular apertures of FSS Layer 1 and FSS Layer 2 are: $a=1.80 \mathrm{~cm}$ (along $X$-axis); and $b=0.55 \mathrm{~cm}$ (along $Y$-axis). The periodicities of the array along $X$ - and $Y$ directions are: $T_{x}=2.325 \mathrm{~cm}$ and $T_{y}=1.555 \mathrm{~cm}$, respectively. Here rectangular lattices are considered, i.e., $\alpha=90^{\circ}$ inside the FSS structure. The polyurethane foam (relative permittivity $\varepsilon_{r}=1.23$, electric loss tangent, $\tan \delta_{e}=0.0024$ ) is taken as a dielectric medium between the FSS layers and inside the aperture regions. The spacing between the FSS layers is optimized to be $0.3 \lambda$. The transmission characteristics of a single layer thick rectangular aperture-type FSS has been validated with reported result at normal incidence for TM polarization as shown in Fig. 3. Here the Floquet modes with index 10, i.e., $10<m, n>-10$, and waveguide mode with index 9, i.e., $p, q \leq 9$, have been used in the computation of single layer FSS. Excellent agreement is observed between the computed and reported results.

As mentioned in Section 3, the same single-layer thick FSS is cascaded to form double-layered FSS structure with polyurethane foam in between the FSS layers and its transmission characteristics are investigated. Further, the number of Floquet and waveguide modes used in the EM analysis of single layer FSS has been considered for the analysis of double-layered FSS structure. Here the computation time taken by 32 -bit operating system with configurations; Intel Core (TM) 


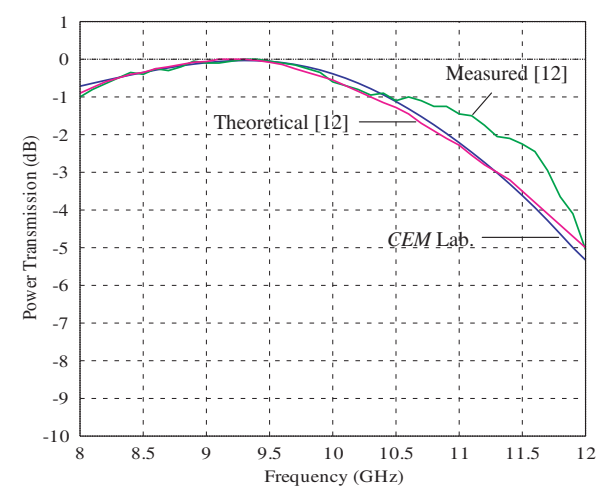

Figure 3. Transmission coefficient of single-layer thick FSS at normal incidence.

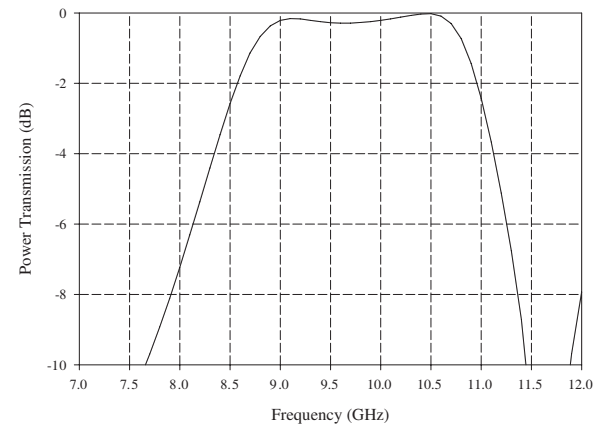

Figure 4. Transmission characteristics of double-layered thick FSS at normal incidence for $d=0.3 \lambda$.

2 Duo CPU and 4 GB RAM is around 1 minute. Fig. 4 shows the power transmission at normal incidence for TM polarization for $d=0.3 \lambda$. It is observed that the double-layered FSS exhibit superior roll-off characteristics outside the band and excellent transmission inside the band. It shows more than $95 \%$ transmission over a frequency range from $8.84 \mathrm{GHz}$ to $10.74 \mathrm{GHz}$. The power transmission characteristics for $d=0.3 \lambda$ at the incidence angle $30^{\circ}$ are also investigated as shown in Fig. 5. It is noted that the bandwidth of the transmission decreases with the incidence angle. Further, the dependence of power transmission characteristics on the spacing between the layers is studied at $30^{\circ}$ as shown in Fig. 6. It is observed that the optimum band-pass response is achieved for $0.3 \lambda$ spacing between the layers. The present FSS structure does not show superior transmission characteristics at high incidence angles $\left(>30^{\circ}\right)$ and hence those results are not included. 


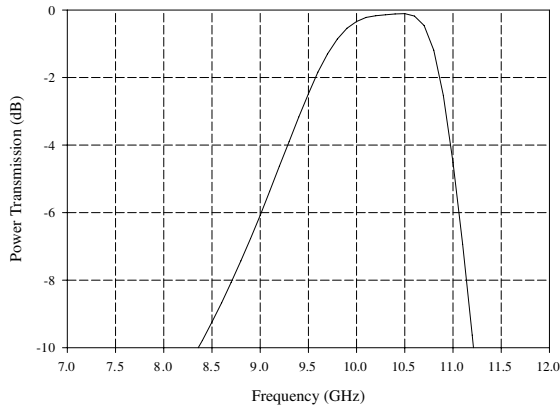

Figure 5. Transmission characteristics of double-layered thick FSS at incidence angle $30^{\circ}$ for $d=$ $0.3 \lambda$.

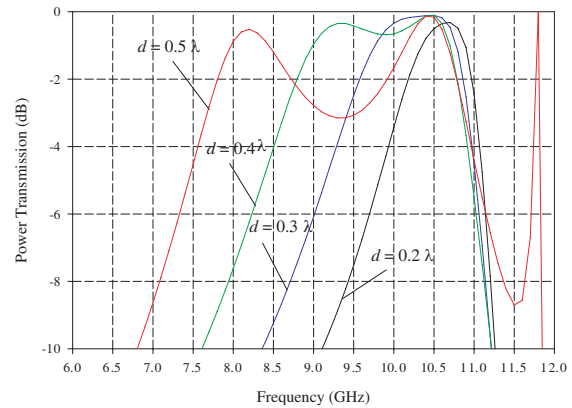

Figure 6. Transmission characteristics of double-layered thick FSS for different spacings between the layers at incidence angle $30^{\circ}$.

\section{CONCLUSIONS}

In the present work, the MM-GSM technique is efficiently used for identifying the modes that contribute to the transmission characteristics of the cascaded thick FSS structure. Further, it provides accurate prediction of EM performance characteristics of cascaded FSS structures with less computational complexity. The cascaded thick FSS structure considered in the present work shows very good inband transmission characteristics and excellent roll-off characteristics outside the band. Moreover, the presence of thick FSS structures on the end- sides of the radome wall configuration offers more structural rigidity (for catering to the extreme aerodynamic stresses), which is an advantage for airborne applications. The present work also shows that the double-layered FSS structure is a suitable choice for the design of spherical and cylindrical airborne radomes.

\section{ACKNOWLEDGMENT}

This work was carried out under the CSIR-sponsored Project FAC-000107 at the CSIR-National Aerospace Laboratories, Bangalore, India.

\section{REFERENCES}

1. Gerini, G. and L. Zappelli, "Phased arrays of rectangular apertures on conformal cylindrical surfaces: A multimode equivalent network approach," IEEE Trans. Antennas Propagat., Vol. 52, No. 7, 1843-1850, 2004. 
2. Gerini, G. and L. Zappelli, "Multilayer array antennas with integrated frequency selective surfaces conformal to a circular cylindrical surface," IEEE Trans. Antennas Propagat., Vol. 53, No. 6, 2020-2030, 2005.

3. Munk, B. A., Frequency Selective Surfaces: Theory and Design, John Wiley, New York, 2000.

4. Lee, S. W., G. Garrillo, and C. Law, "Simple formulas for transmission through periodic metal grids or plates," IEEE Trans. Antennas Propagat., Vol. 30, No. 5, 904-909, 1982.

5. Kipp, R. A. and C. H. Chan, "A numerically efficient technique for the method of moments solution to planar periodic structures in layered media," IEEE Trans. Microwave Theory Tech., Vol. 42, No. 4, 635-643, 1994.

6. Vardaxoglou, J. C., A. Hossainzadeh, and A. Stylianou, "Scattering from two-layer FSS with dissimilar lattice geometries," IEE Proceedings - H, Vol. 140, No. 1, 59-61, 1993.

7. Prakash, V. V. S. and R. Mittra, "An efficient technique for analyzing multiple frequency selective-surface screens with dissimilar periods," Microwave and Optical Technology Letters, Vol. 35, No. 1, 23-27, 2002.

8. Munk, B. A., "Space Filter," U.S. Patent 4125841, 1978.

9. Vardaxoglou, J. C., Frequency Selective Surfaces: Analysis and Design, John Wiley, New York, 1997.

10. Loui, H., "Modal analysis and design of compound gratings and frequency selective surfaces," Ph.D. Thesis, 139, Department of Electrical and Computer Engineering, University of Colorado, Boulder, 2006.

11. Widenberg, B., "A general mode matching technique applied to band-pass radomes," Electromagnetic Theory Tech. Report TEAT-7098, 33, Department of Electroscience, Lund Institute of Technology, Sweden, 2001.

12. Widenberg, B., S. Poulsen, and A. Karlsson, "Thick screens perforated with a periodic array of aperture with arbitrary crosssection," Electromagnetic Theory Tech Report TEAT-7082, 30, Department of Electroscience, Lund Institute of Technology, Sweden, 1999.

13. Wu, T. K., Frequency Selective Surface and Grid Array, John Wiley, New York, 1995. 